\title{
Data-Driven Computational Model to Assess the Risk of Epidemics in Global Mass Gatherings
}

\author{
Sultanah Alshammari ${ }^{* 1,2}$ and Armin Mikler ${ }^{1}$ \\ ${ }^{1}$ University of North Texas, Denton, TX, USA; ${ }^{2}$ King Abdulaziz University, Jeddah, Saudi Arabia
}

\section{Objective}

To develop a computational model to assess the risk of epidemics in global mass gatherings and evaluate the impact of various measures of prevention and control of infectious diseases.

\section{Introduction}

Global Mass gatherings (MGs) such as Olympic Games, FIFA World Cup, and Hajj (Muslim pilgrimage to Makkah), attract millions of people from different countries. The gathering of a large population in a proximity facilitates transmission of infectious diseases [1]. Attendees arrive from different geographical areas with diverse disease history and immune responses. The associated travel patterns with global events can contribute to further disease spread affecting a large number of people within a short period and lead to a potential pandemic. Global MGs pose serious health threats and challenges to the hosting countries and home countries of the participants [2]. Advanced planning and disease surveillance systems are required to control health risks in these events. The success of computational models in different areas of public health and epidemiology motivates using these models in MGs to study transmission of infectious diseases and assess the risk of epidemics. Computational models enable simulation and analysis of different disease transmission scenarios in global MGs. Epidemic models can be used to evaluate the impact of various measures of prevention and control of infectious diseases.

\section{Methods}

The annual event of the Hajj is selected to illustrate the main aspects of the proposed model and to address the associated challenges. Every year, more than two million pilgrims from over 186 countries arrive in Makkah to perform Hajj with the majority arriving by air. Foreign pilgrims can stay at one of the holy cities of Makkah and Madinah up to 30-35 days prior the starting date of the Hajj. The long duration of the arrival phase of the Hajj allows a potential epidemic to proceed in the population of international pilgrims. A Stochastic SEIR (Susceptible-Exposed-Infected-Recovered) agent-based model is developed to simulate the disease transmission among pilgrims. The agent-based model is used to simulate pilgrims and their interactions during the various phases of the Hajj. Each agent represents a pilgrim and maintains a record of demographic data (gender, country of origin, age), health data (infectivity, susceptibility, number of days being exposed or infected), event related data (location, arrival date and time), and precautionary or health-related behaviors.

Each pilgrim can be either healthy but susceptible to a disease, exposed who are infected but cannot transmit the infection, or infectious (asymptomatic or symptomatic) who are infected and can transmit the disease to other susceptibles. Exposed individuals transfer to the infectious compartment after $1 / \alpha$ days, and infectious individuals will recover and gain immunity to that disease after $1 / \gamma$ days. Where $\alpha$ is the latent period and $\gamma$ is the infectious period. Moving susceptible individuals to exposed compartment depends on a successful disease transmission given contact with an infectious individual. The disease transmission rate is determined by the contact rate and the transmission probability per contact. Contact rate and mixing patterns are defined by probabilistic weights based on the features of infectious pilgrims and the duration and setting of the stage where contacts are taking place. The initial infections are seeded in the population using two scenarios (Figure 1) to measure the effects of changing, the timing for introducing a disease into the population and the likelihood that a particular flight will arrive with one or more infected individuals.

\section{Results}

The results showed that the number of initial infections is influenced by increasing the value of $\lambda$ and selecting starting date within peak arrival days. When starting from the first day, the average size of the initial infectious ranges from $0.05 \%$ to $1 \%$ of the total arriving pilgrims. Using the SEIR agent-based model, a simulation of the H1N1 Influenza epidemic was completed for the 35-days arrival stage of the Hajj. The epidemic is initiated with one infectious pilgrim per flight resulting in infected $0.5 \%$ of the total arriving pilgrims. As pilgrims spend few hours at the airport, the results obtained from running the epidemic model showed only new cases of susceptible individuals entering the exposed state in a range of $0.20 \%$ to $0.35 \%$ of total susceptibles. The number of new cases is reduced by almost the same rate of the number of infectious individuals following precautionary behaviors.

\section{Conclusions}

A data-driven stochastic SEIR agent-based model is developed to simulate disease spread at global mass gatherings. The proposed model can provide initial indicators of infectious disease epidemic at these events and evaluate the possible effects of intervention measures and health-related behaviors. The proposed model can be generalized to model the spread of various diseases in different mass gatherings, as it allows different factors to vary and entered as parameters.

\section{Scenario 1}

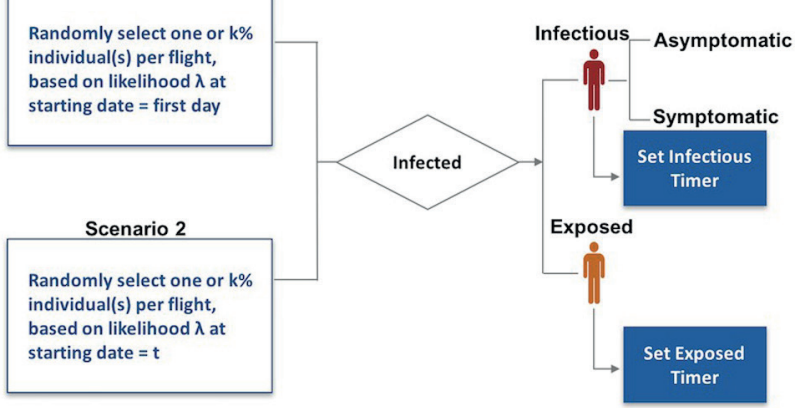

Figure 1: Scenarios to determine the initial number of infected or exposed individuals upon arrival.

\section{Keywords}

mass gatherings; agent-based modeling; infectious diseases; epidemic; Hajj 


\section{References}

1. Memish ZA, Stephens GM, Steffen R, Ahmed QA. Emergence of medicine for mass gatherings: lessons from the Hajj. The Lancet infectious diseases. 2012 Jan 31;12(1):56-65.

2. Chowell G, Nishiura H, Viboud C. Modeling rapidly disseminating infectious disease during mass gatherings. BMC medicine. 2012 Dec $7 ; 10(1): 159$

\section{*Sultanah Alshammari}

E-mail: sultanahalshammari@my.unt.edu 